\title{
Human Blood Cell Sensing with Platinum Black Electroplated Impedance Sensor
}

\author{
Siyang Zheng, Student Member, IEEE, Mandheerej S. Nandra and Yu-Chong Tai, Fellow, IEEE \\ Caltech Micromachining Laboratory, California Institute of Technology, Pasadena, CA, U.S.A.
}

\begin{abstract}
AC impedance sensing is an important method for biological cell analysis in flow cytometry. For micro impedance cell sensors, downsizing electrodes increases the double layer impedance of the metal-electrolyte interface, thus leaves no sensing zone in frequency domain and reduces the sensitivity significantly. We proposed using platinum black electroplated electrodes to solve the problem. In this paper, using this technique we demonstrated human blood cell sensing with high signal to noise ratio.
\end{abstract}

Keywords-impedance sensor; Coulter counter; blood cells; platinum black electroplating

\section{INTRODUCTION}

Electrical impedance sensing has been one of the preferred technologies for particle sensing and counting. Since the invention of Coulter counter by Wallace H. Coulter in 1940s [1], it has been used in bulk blood counters and flow cytometers. The initial DC resistance sensing also extended to AC impedance sensing [2]. For electrical impedance sensing in a flow system, cells flow by sensing electrodes one by one. This creates changes of the electrical impedance between the electrodes. Therefore, the existence of a biological cell and some of its properties can be determined. At low $\mathrm{AC}$ frequency (under $100 \mathrm{kHz}$ ), the signal is determined mainly by the cell volume. At higher frequency $(100 \mathrm{kHz}$ to $10 \mathrm{MHz})$, the intracellular structures also contribute to the overall measured impedance and become explorable measurands [3].

Traditional electrical impedance sensors require large electrode size to reduce the metal-electrolyte interface impedance (i.e. double layer impedance) and allow the particle impedance to dominate the overall system impedance. Bulk blood counters, for example, use macro-electrodes (such as Platinum wire) across the aperture whose size is in the range of hundreds of micron. Recently, there's a push to shrink the sensor size as miniaturization enables better sensitivity, lower coincidence error rate, smaller sample volume and faster detection with parallelization. However, there is a major difficulty associated with micro impedance sensing. The series metal-electrolyte impedance is inversely proportional to the electrode surface area so the downsizing unavoidably reduces the sensor sensitivity [4-7]. For examples, recent works on micro DC Coulter sensors were forced to stay with very large thin film electrode [8], macroscopic gold pin electrodes [9] and salt bridge with nonpolarizing $\mathrm{Ag} / \mathrm{AgCl}$ electrodes [10]. Another approach is to use microelectrodes in high-frequency $\mathrm{AC}$ impedance sensing mode because high frequency reduces the double-layer impedance. Unfortunately there is an upper limit of the operation frequency imposed by the stray capacitance between sensing electrodes, which is resulted from coupling and non-ideal isolation. So to study particles with AC impedance sensing, the device is limited to a frequency range, which is high enough to bypass electrode double layer impedance and low enough that the stray capacitance does not play a significant role in the overall system impedance [7]. Micro AC impedance sensors have been attempted to sense cell suspensions and single cells. The problem of large double layer impedance was overcome by electroplating very thick gold micro electrodes [5] or reducing the stray capacitance to shift the sensing to high frequency range [11]. Generally speaking, high frequency $\mathrm{AC}$ impedance sensing requires more consideration in electrode design, chip isolation, signal conditioning and processing. In this study, we demonstrated Platinum black electroplated electrodes as an effective way to extend AC impedance sensing to lower frequency range. This not only expands the sensing frequency range of micro impedance sensors, but also avoided some of the complexities associated with the high frequency sensing.

Theoretically, electrodes with high effective surface area are ideal to overcome the problem with double layer impedance. Platinum black electroplated electrodes have porous surface structures and thus increase the effective electrode surface area. It can reduce the electrode surface double layer impedance by two orders of magnitude. Consequently the channel impedance becomes the dominant system impedance in some intermediate frequency range and the device become sensitive to what is flowing inside. Platinum black electroplated micro electrodes have been used for extracellular neural signal recording [12-14], gas sensing [15], thermal infrared detection [16]. Specifically, we report here the first use of Platinum black electroplated microelectrodes as AC impedance sensors for human blood cell sensing.

\section{THEORY}

The device can be modeled electrically as the channel impedance $Z$ in series with a constant phase element $(C P E)$ and then in parallel with the stray capacitance $C_{s t}$ (Fig.1). $Z$ includes both the electrolyte impedance and impedance of particle to be sensed. In this paper, the particles were sensed at $10 \mathrm{kHz}$, so the channel impedance was effectively a resistance $R$. $C_{s t}$ is the unwanted coupling capacitance between the two electrodes. $\mathrm{CPE}$ is contributed by the double layer impedance of the metal-electrode interface. Impedance of CPE is $Z_{0} /(j \omega)^{\alpha}$, with constant $Z_{0}$, angular frequency $\omega$ and exponent $\alpha$. The phase of CPE is a constant of - $\alpha \pi / 2$. Normal metal electrodes have $\alpha$ close to one and the CPE behaves like a capacitor with capacitance $l / Z_{0}$ and phase $-\pi / 2$. There are at least two effects that can make $\alpha$ deviate from one. The surface roughness can 
change $\alpha$ from 1 in perfect flat 2D electrodes to 0.5 in $3 \mathrm{D}$ porous cubic electrodes [17]. Platinum black electroplating can greatly change the surface roughness of metal electrodes to transform them from flat 2D surface toward 3D structures. Another effect that is likely to change $\alpha$ in micro domain is the transport of electrochemical active molecules on the electrode surfaces. In micro devices, the ratio of electrode surface area to the volume of the sensing zone is so large that the electrochemical reaction on the electrode surface is diffusion limited. This also reduces $\alpha$

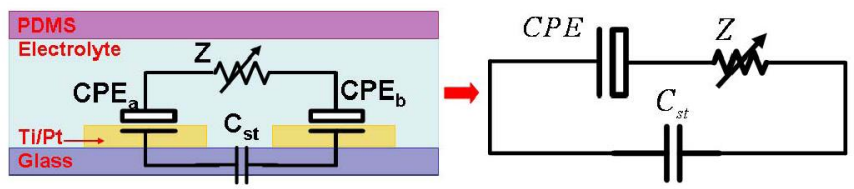

Figure 1. System Model

From the system model, the overall measured impedance can be written as:

$$
Z=\frac{1}{j \omega C_{s t}+\frac{1}{R+Z_{0} /(j \omega)^{\alpha}}}
$$

For channel resistance to dominate in some frequency range, it requires:

$$
R \geq C_{s t}^{\alpha / 1-\alpha} Z_{0}^{1 / 1-\alpha}
$$

Clearly from equation (2), lowering the stray capacitance and double layer impedance are favored for particle sensing. If the equation (2) is satisfied, the system shows some typical asymptotic behaviors. When $\omega$ approaches zero, the system is reduced to the channel impedance in series with the CPE. When $\omega$ approaches infinity, the system approaches the channel impedance in parallel with the stray capacitance. These asymptotic behaviors are illustrated in the Nyquist plot of Fig.2, which can be used to extract parameters from measurement.

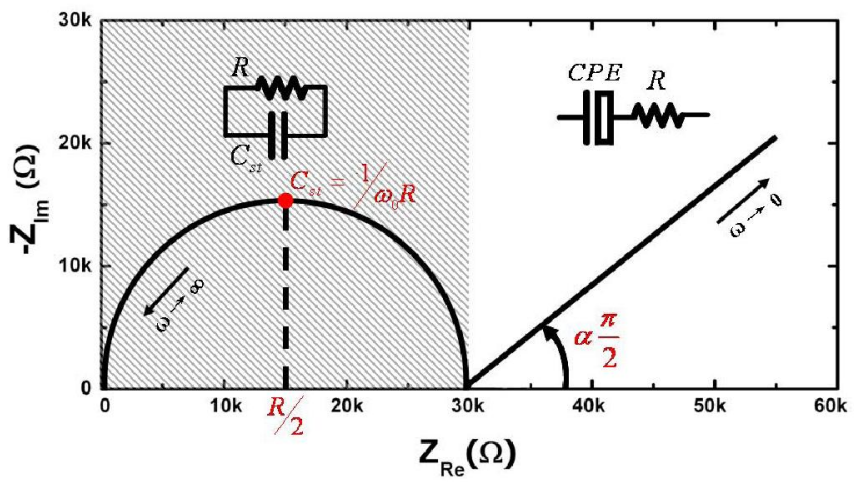

Figure 2. Asymptotic behaviors in Nyquist plot. $\omega_{0}$ is the angular frequency when $Z_{R e}$ is $R / 2$.

In order for the system to sense flowing-by particles, the particle impedance should be the major component in overall system impedance at the excitation frequency. This is difficult to achieve in micro systems because the miniaturization of the electrodes. In low frequency range, impedance of CPE dominates the system impedance, while at high frequency the stray capacitance in parallel to the system dominates. To enable particle sensing in low frequency domain, an effective way is to lower impedance of CPE by platinum black electroplating. It can increase surface area by two orders of magnitude and thus reduce electrical impedance proportionally.

\section{DESIGN AND FABRICATION}

The chip layout is illustrated as Fig.3(A). Two fluidic chambers are separated by an aperture. The width of the aperture is $15 \mu \mathrm{m}$. The length of the aperture is $30 \mu \mathrm{m}$. One pair of metal electrodes senses the electrical impedance across the aperture. The size of the chamber is very large compared with the aperture so that the measured channel impedance is dominated by the impedance of the aperture region. The distance of the electrodes is $70 \mu \mathrm{m}$. At the inlet and outlet, some filter structures are designed to mechanically block contaminants and particle aggregates and preventing them from clogging the aperture. Channel height is optimized to be around $15 \mu \mathrm{m}$ so that all blood cells can pass while keeping signal magnitude as high as possible.

The device fabrication was started with molding channel structure on PDMS with DRIE etched $\mathrm{Si}$ mold. Ti/Pt $(200 \AA / 2500 \AA)$ electrodes were patterned on soda lime glass substrate with lift-off process. Platinum black was then electroplated to the selected areas Fig.3(B). Electroplating solution contains $1 \%$ chloroplatinic acid, $0.0025 \%$ hydrochloric acid, and $0.01 \%$ lead acetate in water [13]. Electrodes were plated with $5 \mathrm{~V}$ DC through a $10 \mathrm{M} \Omega$ series resistor for up to four minutes to achieve maximal double layer impedance reduction. The thickness of the electroplated platinum black layers was normally larger than $1 \mu \mathrm{m}$. Finally the PDMS block was bonded to glass surface at $80^{\circ} \mathrm{C}$ overnight. After fabrication, the chip was glued and wire bonded onto a printed circuit board (PCB).

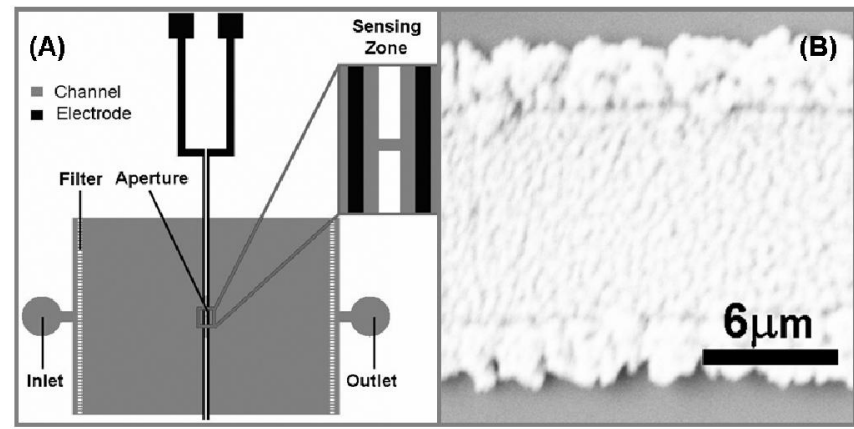

Figure 3. Device design layout (A) and SEM of Platinum black electroplated electrodes $(B)$.

\section{RESULTS}

The effectiveness of platinum black electroplating was studied by measuring the impedance spectra with LF impedance analyzer (HP 4192A) before and after platinum black electroplating on devices filled with electrolyte phosphate buffered saline (PBS). Fig. 4 and Fig.5 are the Bode and the Nyquist plot of simulated and measured system impedance data. Before platinum black electroplating, the system impedance response shows capacitive characteristics in all the frequency range. And the impedance responses in air, 
DI water and PBS have no difference (data no shown here). After Platinum black electroplating, resistive characteristic in the frequency range from $100 \mathrm{~Hz}$ to $3 \mathrm{MHz}$ (the plateau in the figure) showed up. This is where the channel resistance dominates and it is the frequency range suitable for cell sensing. CPE parameter $\alpha$ was determined to be around 0.5 from fitting the data to both plots, which indicated extreme surface roughness after Platinum black electroplating and the surface seemed to be $3 \mathrm{D}$ in electrochemical sense.

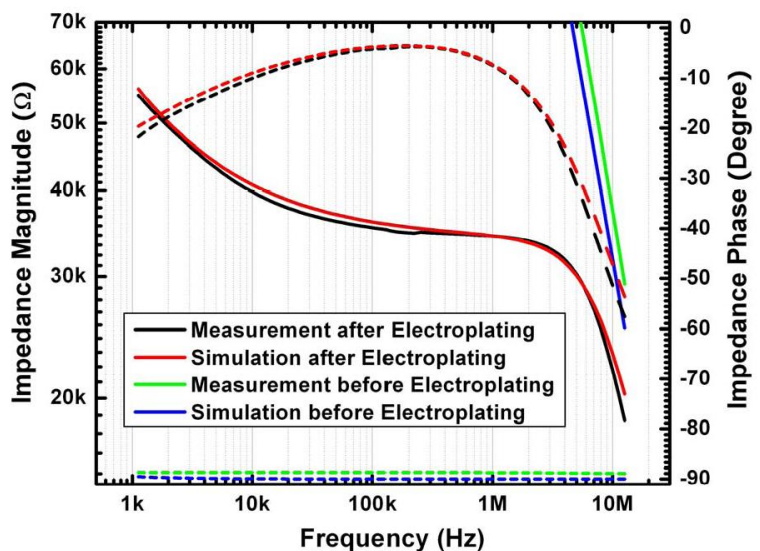

Figure 4. Bode plot of simulated and measured system impedance magnitude (solid lines) and phase (dash lines) response in electrolyte PBS. Data were fitted by least-squares curve-fitting, with parameters $Z_{0}=2.24 \mathrm{M} \Omega$, $\mathrm{C}_{\mathrm{st}}=0.5 \mathrm{pF}$ and $\alpha=0.5$.

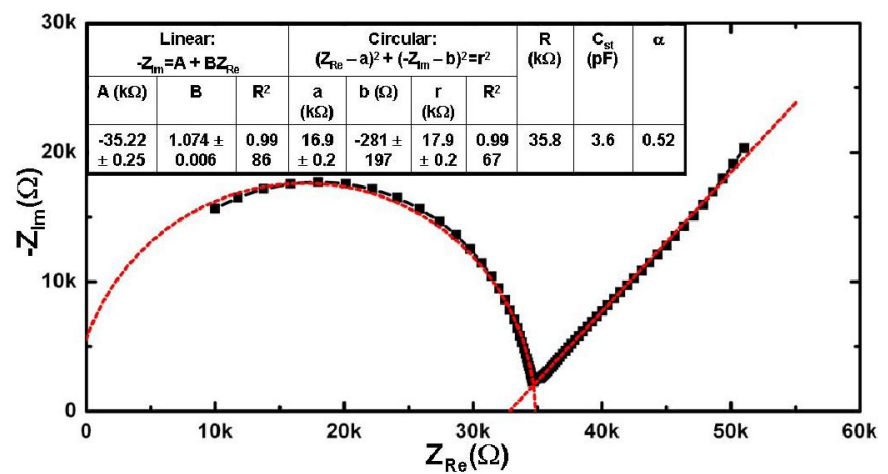

Figure 5. Nyquist plot of measured impedance data. The table contains parameters obtained by fitting Nyquist plots to asymptotes. $\mathrm{R}^{2}$ is the multiple correlation coefficient.

For particle sensing, the sample was pumped into device with a syringe pump. The system was excited with $100 \mathrm{mV}$ sinusoidal wave at $10 \mathrm{kHz}$. Output signal was amplified with a lock-in amplifier. Data was recorded with a deep memory oscilloscope and then transferred into a computer. The peaks were detected with a Matlab program.

The particle sensing was first calibrated with polystyrene beads with different sizes. The flow rate was $1 \mathrm{~nL} / \mathrm{min}$. The particle concentrations were in the range of $10^{4} / \mu \mathrm{L}$. Fig. 6 shows a time trace and a typical peak of $8 \mu \mathrm{m}$ polystyrene beads. The peak height was $0.2608 \mathrm{~V} \pm 0.0127 \mathrm{~V}$, which meant under $5 \%$ standard deviation. The duration of the peaks was $17.21 \mathrm{~ms} \pm 0.67 \mathrm{~ms}$, which corresponded well with the expected time that the beads passed the sensing zone. We also measured signals from $5 \mu \mathrm{m}$ and $10 \mu \mathrm{m}$ polystyrene beads as plotted in Fig.7. Theoretical channel resistance change under DC excitation was calculated and plotted as the dash line. The measured bead resistance changes fit well on the curve. It is well know as Coulter principle that under DC excitation the signal magnitude is determined by the size of the particles. Our measurement results confirmed that for $10 \mathrm{kHz}$ excitation frequency, the magnitude of resistance change was still determined by the size of the particles.



Figure 6. Sensing of $8 \mu \mathrm{m}$ beads tested at $10 \mathrm{kHz}$. (A) a time trace. (B) a typical peak. The solid line is the least-squares curve-fitting to parabola.

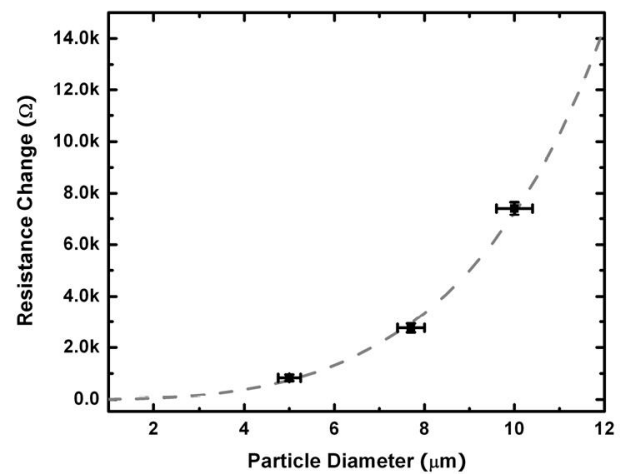

Figure 7. Sensing signal depends on the size of polystyrene beads. Dash line is theoretical relationship at DC.

Human blood cell sensing was performed with either diluted whole blood or diluted leukocyte rich plasma. Whole blood samples were obtained from healthy donors and used within 48 hours. Normal whole blood has an erythrocyte to leukocyte ratio about one thousand to one. So it is good for erythrocyte characterization. Leukocyte rich plasma was prepared with Wintrobe method. Erythrocyte to leukocyte ratio can be reduced by two orders of magnitude. So it is better for leukocyte testing. Fig. 8 shows a time trace and some example peaks for human whole blood diluted by one thousand times. The signal to noise ratio was not as good as the $8 \mu \mathrm{m}$ beads and slowly drifting of baseline was sometimes experienced. The distribution of pulse height matched well with the known volume distribution of erythrocytes (Fig.9). Leukocyte rich plasma was used for leukocyte sensing. The tail part of the peak height distribution was contributed by leukocytes. It resembles the known leukocytes volume distribution (Fig.10).

\section{CONCLUSION AND Discussion}

We demonstrated sensing of human erythrocytes and leukocytes with platinum black electroplated micro impedance sensors with high signal to noise ratio. The advantages of downsizing electrodes and the sensing zone in micro devices 
include increased sensitivity, reduced coincidence errors, lowered sample dilution factor and thus increased system throughput.

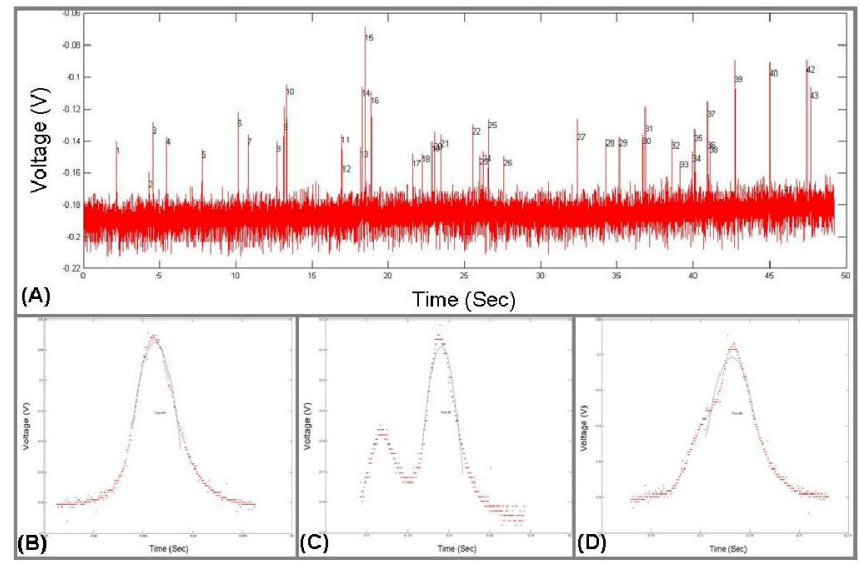

Figure 8. Sensing cells in diluted human whole blood. (A) A time trace. (B) A typical peak. (C) Two close peaks. (D) Two peaks too close to be seperated. The solid lines are least-squares curve-fittings to parabola.



Figure 9. Pulse height distribution of diluted human whole blood, in which leukocyte to erythrocyte ratio is about one to a thousand. Inset is erythrocyte volume distribution from [18].



Figure 10. Pulse height distribution of leukocyte rich plasma, in which leukocyte to erythrocyte ratio is about one to ten. Middle inset shows the detail of the tail of the distribution. Top inset is leukocyte volume distribution [18].

\section{ACKNOWLEDGMENT}

This work is supported by the National Space Biomedical Research Institute through NASA NCC 9-58. The authors would like to thank other members of the Caltech Micromachining Laboratory for their valuable assistance.

\section{REFERENCES}

[1] W. H. Coulter, U. S. Patent 2656508, U.S.A. 1953

[2] W. H. Coulter and W. R. Hogo, U. S. Patent 3502974, Ed. U.S.A., 1970.

[3] K. Asami, Progress In Polymer Science, vol. 27, pp. 1617-1659, 2002.

[4] S. Zheng, M. Liu, H. L. Kasdan, and Y. C. Tai, IEEE-NEMS'06: Zhuhai, China, 2006.

[5] H. E. Ayliffe, A. B. Frazier, and R. D. Rabbitt, Journal Of MEMS, vol. 8, pp. 50-57, 1999.

[6] S. Gawad, K. Cheung, U. Seger, A. Bertsch, and P. Renaud, Lab On A Chip, vol. 4, pp. 241-251, 2004.

[7] J. H. Nieuwenhuis, F. Kohl, J. Bastemeijer, P. M. Sarro, and M. J. Vellekoop, Sensors and actuators. B, Chemical, vol. 102, pp. 44, 2004.

[8] D. Satake, H. Ebi, N. Oku, K. Matsuda, H. Takao, M. Ashiki, and M. Ishida, Sensors and actuators. B, Chemical, vol. 83, pp. 77, 2002.

[9] D. W. Lee, S. Yi, and Y.-H. Cho, MEMS2005, Miami Beach, Florida, USA, 2005.

[10] H. G. Chun, T. D. Chung, and H. C. Kim, Analytical Chemistry, vol. 77, pp. 2490-2495, 2005.

[11] S. Gawad, L. Schild, and P. Renaud, Lab on a chip, vol. 1, pp. 76, 2001.

[12] D. R. Jung, D. S. Cuttino, J. J. Pancrazio, P. Manos, T. Cluster, R. S. Sathanoori, L. E. Aloi, M. G. Coulombe, M. A. Czamaski, D. A. Borkholder, G. T. A. Kovacs, P. Bey, D. A. Stenger, and J. J. Hickman, Journal Of Vacuum Science \& Technology A, vol. 16, pp. 1183-1188, 1998 .

[13] M. P. Maher, J. Pine, J. Wright, and Y. C. Tai, Journal Of Neuroscience Methods, vol. 87, pp. 45-56, 1999.

[14] H. Oka, K. Shimono, R. Ogawa, H. Sugihara, and M. Taketani, Journal Of Neuroscience Methods, vol. 93, pp. 61-67, 1999.

[15] C. A. Marrese, Analytical Chemistry, vol. 59, pp. 217-218, 1987.

[16] W. Lang, K. Kuhl, and H. Sandmaier, Sensors And Actuators A-Physical, vol. 34, pp. 243-248, 1992.

[17] W. H. Mulder, J. H. Sluyters, T. Pajkossy, and L. Nyikos, Journal Of Electroanalytical Chemistry, vol. 285, pp. 103-115, 1990.

[18] S. B. McKenzie, Clinical Laboratory Hematology: Prentice Hall, 2004. 